\title{
Two-Tier WBAN/WLAN Healthcare Networks; Priority Considerations
}

\author{
Saeed Rashwand \\ Department of Computer Science \\ University of Manitoba
}

\author{
Jelena Mišić \\ Department of Computer Science \\ Ryerson University
}

\begin{abstract}
In this work, by bridging the IEEE 802.15.6-based WBANs and the IEEE 802.11e EDCA-based WLAN we develop a wireless healthcare network. We assign 8 WBAN User Priorities (UPs) to the WBAN nodes. By mapping the UPs into 4 WLAN Access Categories (ACs) we convey the medical data to the WLAN access point. We investigate the network performance under varying priority differentiation and number of nodes in the WLAN. We study the impacts of AC differentiation by Arbitrary Inter-Frame Space (AIFS) and Contention Window (CW) on the performance of WBAN and regular WLAN nodes. The results of this work indicate that differentiation by AIFS is more appropriate to preserve relative order of frame response times established in WBAN.
\end{abstract}

\section{INTRODUCTION}

A Wireless Body Area Network (WBAN) is a body monitoring network which aims to predict and diagnose any diseases and monitor the response of the body to treatments. The WBAN includes small and intelligent sensors to pervasively and ubiquitously monitor the human body's health condition. The sensors produce the medical data and transfer to the WBAN hub which operates as the WBAN's coordinator. The hub collects the medical data from the the nodes. The collected medical data must be transferred to a central medical server for further processing and storage.

In this work, we deploy WLAN for transferring the medical data from the WBANs to the central medical server. Since, WBANs and WLANs operate under two different wireless communication standards we need to bridge WBANs and WLAN in order to build the wireless healthcare network. Due to the specific characteristics of the WBANs, the IEEE 802.15.6 standard was introduced for shortrange, low power, and reliable wireless communications around or in the human body [1]. Hence, we adopt the IEEE 802.15.6 standard for the patient's body network (WBAN). We select IEEE 802.11e EDCA for the second hop to convey the medical data to the WLAN access point because it supports user priorities in the network and provides higher transmission opportunities for higher priority data.provides enough tools to preserve the WBAN priorities [2]. We define the WBAN/WLAN bridge as a node which operates in both WBAN and WLAN. The bridge operates as the WBAN hub collecting the medical data from the WBAN nodes. It also operates as a station in the WLAN transferring the medical data to the WLAN access point. Since AP and medical server have wired connection, we will neglect delays over that hop. In Fig. 1 networking structure of a healthcare system including the WBANs, bridges, and the WLAN is depicted.

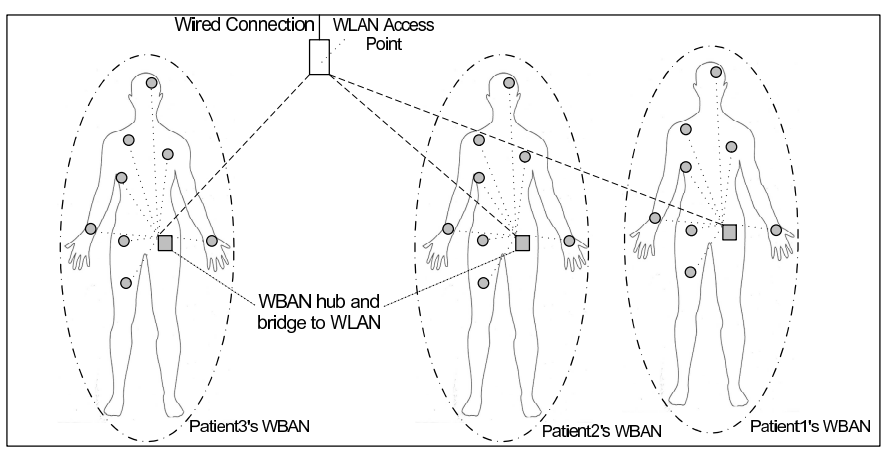

Fig. 1. Networking structure of two hop healthcare wireless network

In our previous work, we separately developed detailed and complex analytical models for IEEE 802.15.6 and IEEE 802.11e EDCA [3], [4]. We validated the analytical models with simulation models. The frame arrival process in the bridges follows a general distribution due to the characteristics of output process from sensing nodes. This would lead to a $\mathrm{G} / \mathrm{G} / 1$ queuing model in the bridges which significantly complicates the analytical modelling [5]. Therefore, in this work we deploy simulation modelling for the WBAN/WLAN bridged network.

There is a small number of studies in the literature which investigate the performance of the IEEE 802.15.6 standard by developing simulation and/or analytical models [6], [7], [3]. The developed models in [3] are the most advanced ones in the literature which consider 8 UPs, Access Phases, and detailed CSMA/CA mechanism of the standard. There are more studies in the literature to evaluate the performance of IEEE 802.11e EDCA standard models such as [8], [9], [4], [10]. Though the IEEE 802.15.6 and IEEE 802.11e EDCA standards are separately studied through analytical and/or simulation models there is no work in the literature to investigate the performance of their interconnection.

Bridging of WBANs and WLAN is challenging since the MAC parameters of the IEEE 802.15.6 and the IEEE 802.11e EDCA standards have strong impact on the performance of the network. In the WBAN, the access phases lengths are con- 
figurable but $\mathrm{CW}_{\min }$ and $\mathrm{CW}_{\max }$ differentiation parameters are constant according to IEEE 802.15.6. However, all four differentiation parameters of IEEE 802.11e EDCA $\left(\mathrm{CW}_{\text {min }}\right.$, $\mathrm{CW}_{\max }$, AIFS, and TXOP) are configurable.

In this work, we develop a healthcare network in which there are all the 8 WBAN UPs and 4 WLAN ACs. The medical data streams are assigned to different UPs according to their priorities. We bridge the WBANs and WLAN by mapping the WBAN UPs into WLAN ACs. The WLAN includes the WBAN/WLAN bridges and regular WLAN nodes. WLAN nodes generate data frames in all ACs. For the developed healthcare network, we investigate the impact of AC differentiation by AIFS and CW on the performance under varying number of nodes and frame arrival rates for regular WLAN nodes.

The remainder of this paper is organized as follows: Section II addresses the bridging mechanism between WBANs and WLAN. In Section III we investigate the WBAN/WLAN bridges' and the regular WLAN nodes' performance for the cases when the AC differentiation is performed through either AIFS or CW. Finally, Section IV concludes the paper summarizing the findings of the study.

\section{BRIDGING WBANS AND WLAN}

We deploy the IEEE 802.15.6 standard for wireless communication in the WBANs. Minimum and maximum Contention Window sizes $\left(\mathrm{CW}_{\min }\right.$ and $\left.\mathrm{CW}_{\max }\right)$ differentiate the 8 User Priorities (UPs) in a WBAN, as shown in Table I. The time is divided into beacon periods (superframes) by the hub as shown in Fig. 2. In this work, we assume non-zero values for EAP1 (Exclusive Access Phase) and RAP1 (Random Access Phase) while the lengths of the other access phases are set to zero. For a detailed discussion about the CSMA/CA mechanism and the access phases of the IEEE 802.15.6 standard please refer to [3].

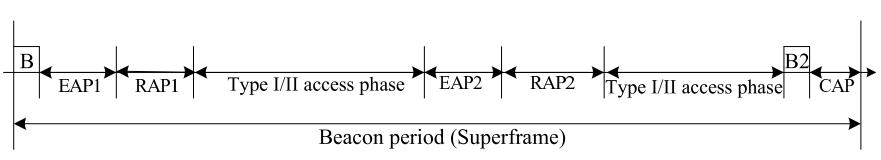

Fig. 2. Layout of access phases in a superframe

IEEE 802.11e EDCA function allows traffic differentiation and user priorities for the stations in the network. EDCA delivers traffic based on differentiating 8 UPs mapped into 4 Access Categories (ACs). The differentiation is achieved by varying the four differentiation parameters; Amount of time a station senses the channel to be idle before backoff or transmission (Arbitrary Inter-Frame Space - AIFS), the length of contention window for backoff $\left(\mathrm{CW}_{\min }\right.$ and $\left.\mathrm{CW}_{\max }\right)$, and the duration of time when a station may transmit after it acquires the channel (TXOP). Each AC has its own queue and channel access differentiation parameters.

We assume that the bridges (hubs) are equipped with two network interfaces. To improve the WBANs performance we assume that the WBAN nodes operate on the non-ISM frequency range of $2360-2400 \mathrm{MHz}$. Thus, they avoid the contention in the $2.4 \mathrm{GHz}$ ISM band (which decreases their interference with the other wireless networks) and achieve the highest possible data frame transmission rate, $971 \mathrm{kbps}$. To avoid interference with the neighbouring WBANs, we assume that the WBANs operate in different frequency channels. The MAC differentiation parameters of the WBANs and WLAN and UPs/ACs mappings are shown in Table I. TXOP value is set to $5000 \mu \mathrm{sec}$ for all ACs in the network.

In this work, we assume that every four data frames with specific UPs are aggregated into a single WLAN data frame. We map the WBAN UPs into WLAN ACs based on the priorities of the WBAN data frames and traffic rates of all UPs, as shown in Table I. We do not use RTS/CTS mechanism for accessing the medium in WBANs since the control frame transmission times are similar to the data frames transmission time. However, we use the RTS/CTS mechanism for accessing the wireless medium in the WLAN because the aggregated data frames are large and the transmission times of the WLAN control frames are much smaller than the data frames transmission times.

\section{Performance Evaluation}

In this section, we evaluate the performance of wireless communications in the healthcare network in which the WBANs and the WLAN create a two-tier network. The WBANs consist of 20 WBAN nodes which are divided into 8 WBAN UPs as shown in Table II. The retransmission limit is set to 7 for all UPs. We set the EAP1 length to $0.1 \mathrm{sec}$ while RAP1 length is set to $0.5 \mathrm{sec}$. In the WBANs, the control frames and headers are transmitted at $91.4 \mathrm{kbps}$ (Kb per sec) while we assume that the data frame payloads are transmitted at $971.6 \mathrm{kbps}$.

The WLAN data frame payloads and the headers are transmitted with the transmission rate of $5.5 \mathrm{Mbps}$ and $1 \mathrm{Mbps}$, respectively. The retransmission limit in the WLAN is also set to 7 for all ACs. In this work, we set the TXOP value to $5000 \mu \mathrm{sec}$, which accommodates any aggregated WBAN data frame or WLAN .

We deploy four performance descriptors for investigating the performance of the network including mean medical data frame access time, mean data frame response time, successful medium access probability, and mean number of successfully transmitted frames during a TXOP access. The performance metrics are presented for two sets of nodes in the network; the WBAN/WLAN bridges and the regular WLAN nodes. The medical data frame arrival to the WBAN nodes follows the Poisson distribution with the mean arrival rates shown in Table II. Though the WBAN nodes' data frame inter-arrival times are exponentially distributed, their arrivals to the hub (bridges) do not follow the Poisson distribution, instead they follow output process from sensing nodes.

We assume that the WBAN nodes operate over a Ricianfaded channel. A data frame's Bit Error Rate (BER) on 
TABLE I

MAPPING WBAN UPs to WLAN ACs (DifFERENTIATION By AIFS:CW min $_{2}=31 \& \mathrm{CW}_{\max }=1023$. DifFERENTIATION By CW:AIFS=2)

\begin{tabular}{|c|c|c|c|c|c|c|c|c|}
\hline \multirow{2}{*}{\multicolumn{4}{|c|}{ WBAN }} & \multicolumn{5}{|c|}{$\begin{array}{l}\text { WLAN } \\
\text { Diff }\end{array}$} \\
\hline UP & & & & $\mathrm{AC}$ & Traffic designation & $\frac{\text { Diff. by AIFS }}{\text { AIFS }}$ & $\begin{array}{r}\text { Diff. } \\
\text { CWmin }\end{array}$ & $\frac{\mathrm{CW}}{\mathrm{CWmax}}$ \\
\hline 0 & Background & 16 & 64 & 0 & Porkonnd & 7 & 63 & 1023 \\
\hline 1 & Best effort & 16 & 32 & 0 & Background & 1 & 03 & 1023 \\
\hline 2 & Excellent effort & 8 & 32 & 1 & Bect effort & 5 & 31 & 511 \\
\hline 3 & Controlled load & 8 & 16 & 1 & Best enort & 5 & 31 & 511 \\
\hline 4 & Video & 4 & 16 & 2 & Videe & 3 & 15 & 255 \\
\hline 5 & Voice & 4 & 8 & 2 & Video & 3 & 15 & 255 \\
\hline 6 & Media data or network control & 2 & 8 & 3 & Voice & 2 & 7 & 127 \\
\hline 7 & Emergency & 1 & 4 & 3 & voice & 2 & 1 & 121 \\
\hline
\end{tabular}

TABLE II

HEALTHCARE NODES ARE SPREAD INTO 8 UPS (NN: NUMBER OF NODES, TL: TRAFFIC LOAD PER PACKET, PS: PAYLOAD SIZE, AC: MAPPED INTO ACCESS CATEGORY)

\begin{tabular}{|c|c|c|c|c|c|}
\hline UP & Node & NN & TL & PS & AC \\
\hline \hline 7 & ECG,EEG & 1,1 & $2,2 \mathrm{p} / \mathrm{s}$ & $150,150 \mathrm{~B}$ & \multirow{2}{*}{3} \\
\hline 6 & EEG & 2 & $2 \mathrm{p} / \mathrm{s}$ & $150 \mathrm{~B}$ & \\
\hline 5 & EEG,Blood Pressure & 1,1 & $2,2 \mathrm{p} / \mathrm{s}$ & $150,150 \mathrm{~B}$ & \multirow{2}{*}{2} \\
\cline { 1 - 5 } 4 & Glucose,Oxygen Saturation,Temperature,Respiration Rate & $1,1,1,1$ & $1,1,1,1 \mathrm{p} / \mathrm{s}$ & $50,50,50,50 \mathrm{~B}$ & \\
\hline 3 & Physical Activity & 2 & $2 \mathrm{p} / \mathrm{s}$ & $50 \mathrm{~B}$ & \multirow{2}{*}{1} \\
\cline { 1 - 5 } 2 & EMG & 2 & $2 \mathrm{p} / \mathrm{s}$ & $500 \mathrm{~B}$ & \\
\hline 1 & ECG & 2 & $2 \mathrm{p} / \mathrm{s}$ & $150 \mathrm{~B}$ & \multirow{2}{*}{0} \\
\hline 0 & EEG & 4 & $1 \mathrm{p} / \mathrm{s}$ & $300 \mathrm{~B}$ & \\
\hline
\end{tabular}

a Rician-faded channel is a function of channel quality, antenna diversity order, and Signal to Noise Ratio (SNR) values for all UPs. We deploy the QPSK modulation scheme for the WBAN nodes to achieve the highest possible data frame transmission rate. We calculate BER of QPSK in Rician-faded channels for different UPs according to [11]. According to the positions, types, and functionalities of healthcare nodes we set the Rician factors for different UPs as: $\left(K_{0}, K_{1}, K_{2}, K_{3}, K_{4}, K_{5}, K_{6}, K_{7}\right)=$ $(1.5,4,3,3,2.5,1.5,1.5,4)$. The larger Rician factor indicates a better channel quality and causes smaller BER. The diversity order, $L$, is set to 1 for all UPs. We obtain the average BERs for all UPs as shown in Table III.

TABLE III

BER VALUES FOR UPS IN WBANS

\begin{tabular}{|c|c|c|c|}
\hline $\mathrm{BER}_{0}, \mathrm{BER}_{5}, \mathrm{BER}_{6}$ & $\mathrm{BER}_{1}, \mathrm{BER}_{7}$ & $\mathrm{BER}_{2}, \mathrm{BER}_{3}$ & $\mathrm{BER}_{4}$ \\
\hline $13.95866^{*} 10^{-5}$ & $2.31524 * 10^{-5}$ & $5.0085 * 10^{-5}$ & $7.21^{*} 10^{-5}$ \\
\hline
\end{tabular}

We deploy the Opnet simulator [12] for modelling the wireless communications in the bridged healthcare network. We developed the simulation model for the IEEE 802.15.6 standard since the Opnet simulator does not have a module with implementation of this standard. In addition, we edited the implemented IEEE 802.11e EDCA in Opnet to follow the standard. The simulation models follow the standards in detail. Finally, we developed the two-tier WBAN/WLAN healthcare network model based on the developed IEEE 802.15.6 and IEEE 802.11e EDCA models.

We consider two different cases where the number of regular WLAN nodes is either 3 or 10 , where generate data frames of all ACs with the payload size of 100B. The WLAN contains 10 WBAN/WLAN bridges. Frame inter-arrival times of regular nodes are exponentially distributed with the mean values shown in the horizontal axis of the plots. We set the TXOP value to $5000 \mu \mathrm{sec}$ for all ACs. Data frames' BER in the WLAN is set to a constant value of $2 * 10^{-5}$.

In this paper, we only display the results when all the network nodes are stable. When a node's performance descriptors leave the linear regime the results are undesirable. The network is unstable when a node in the network is unstable. Hence, we avoid to display the results when a traffic class in a node becomes close to the saturation region. Fig. 3 includes 8 plots for the medical data frame access time in the WBAN/WLAN bridged healthcare network. This time includes two periods. The first period is the time interval between the moment when the medical data frame in the WBAN is generated and the moment when it is successfully transmitted to the hub. The second period is the time interval from the instant when the aggregated WBAN data frame is created in the bridge until the moment when the frame is successfully transmitted to the WLAN access point. The plotted medical data frame access time does not include the individual data frames' queuing delay in the bridges. We show the results for the cases when the number of regular WLAN nodes (3 and 10) and the type of AC differentiation (AIFS differentiation and CW differentiation) vary. In Fig. 3 for all regular WLAN nodes' traffic rates, the time differences between values for pairs of $\mathrm{UP}_{0}$ and $\mathrm{UP}_{1}, \mathrm{UP}_{2}$ and $\mathrm{UP}_{3}, \mathrm{UP}_{4}$ and $\mathrm{UP}_{5}$, and finally $\mathrm{UP}_{6}$ and $\mathrm{UP}_{7}$ are constant which match the UPs' mean frame response time differences in the WBAN.

The plots in Fig. 3 show that when there are 3 regular WLAN nodes in the network, differentiation by CW or AIFS does not considerably affect the response time of the bridges' 
data frames. However, when 10 regular WLAN nodes operate in the network the AC differentiation by AIFS considerably outperforms the differentiation by CW. Small contention window sizes increase the collision probability for the contending nodes and causes transition to early saturation for the CSMA/CA-based wireless networks. Figs. 3(c) and 3(d) show that the mean medical data frame access time for the lowest priority $\mathrm{AC}\left(\mathrm{UP}_{0}\right.$ and $\left.\mathrm{AC}_{0}\right)$ exceeds $0.25 \mathrm{sec}$ where the regular WLAN nodes' data frame arrival rate is approximately equal to $14 \mathrm{fps}$ and $12 \mathrm{fps}$ for the AC differentiation by AIFS and $\mathrm{CW}$, respectively.

Fig. 4 depicts the mean response time of the regular WLAN nodes' data frames in the WLAN when there are 10 bridges and either 3 or 10 regular WLAN nodes and AC differentiation is done through AIFS or CW. The time indicates the duration between the moment when the frame is created in the regular WLAN node until the moment when it is successfully transmitted. The results indicate that the IEEE 802.11e EDCA mechanism is very sensitive to the network traffic load in case of small contention window sizes. Small contention window sizes cause larger data frame access delay when there are a large number of contending nodes in the network. AC differentiation through AIFS improves the data frame response time for the bridges and regular WBAN nodes since it provides moderate differentiation.

Fig. 5 and Fig. 6 depict the successful medium access probability of bridges and regular nodes in the WLAN, respectively. The plots indicate that deploying $\mathrm{AC}$ differentiation in an EDCA-based WLAN by CW increases the collision probability because of small contention window sizes. The larger collision probability would result in larger data frame access time, causing larger end-to-end delay for the medical data frames. At some points $\mathrm{AC}_{1}$ has higher successful medium access probability than $\mathrm{AC}_{2}$ due to the larger number of $\mathrm{AC}_{2}$ nodes compared to the number of $\mathrm{AC}_{1}$ nodes, which causes higher collision probability.

Figs. 6(b) and 6(d) indicate that if $\mathrm{CW}$ is used for $\mathrm{AC}$ differentiation the higher priority ACs experience smaller successful medium access probability because of smaller contention windows. However, since larger numbers of bridges belong to $\mathrm{AC}_{0}$ and $\mathrm{AC}_{2}$, compared to $\mathrm{AC}_{1}$ and $\mathrm{AC}_{3}$ respectively, $\mathrm{AC}_{0}$ and $\mathrm{AC}_{2}$ have smaller medium access probabilities than $\mathrm{AC}_{1}$ and $\mathrm{AC}_{2}$, respectively, as shown in Figs. 5(b) and 5(d).

Fig. 7 represents the mean number of transmitted data frames during the TXOP access for bridges in the WLAN. It indicates that larger number of regular WLAN nodes causes larger number of data frame transmissions within TXOP periods for bridges. According to Figs. 7(a) and 7(b), when there are smaller number of regular WLAN nodes in the network, under low to moderate traffic loads, AC differentiation by $\mathrm{CW}$ causes smaller number of transmissions during the TXOP periods compared to the case when AIFS is used for differentiation. However, when there is larger number of regular WLAN nodes (10 nodes) the AC differentiation by $\mathrm{CW}$ causes larger number of data frame transmissions during the TXOP periods due to higher contention on the medium. In addition, under low to moderate traffic $\mathrm{AC}_{1}$ and $\mathrm{AC}_{2}$ attempt the highest and the smallest numbers of transmissions during TXOP periods because they have the largest and smallest average data frame sizes, respectively.

Fig. 8 shows the mean number of transmitted data frames during TXOP period for regular WLAN nodes. Since the generated traffic rate and data frame sizes by all ACs and the channel quality are identical for all ACs, the higher priority AC transmit smaller number of data frames in TXOP peiods. Fig. 8 indicates that in case of small number of regular WLAN nodes the AC differentiation by AIFS and CW does not significantly affect the number of frame transmissions during TXOP period. In case of 10 regular WLAN nodes, under moderate traffic, there is a large difference between the numbers of frame transmissions during TXOP period for AC differentiation by AIFS and CW (for all regular WLAN nodes' ACs). The difference originates from the fact that smaller contention windows results in higher collision probability and more medium access attempts.

TABLE IV

SUCCESSFUl Frame TRANSMISSION RATE WITHIN TXOP PERIOD IN THE WLAN

\begin{tabular}{|c|c|c|c|c|}
\hline & $\mathrm{AC}_{0}$ & $\mathrm{AC}_{1}$ & $\mathrm{AC}_{2}$ & $\mathrm{AC}_{3}$ \\
\hline bridges & 0.83717 & 0.80189 & 0.90865 & 0.88174 \\
\hline Regular WLAN nodes & 0.95349 & 0.95349 & 0.95349 & 0.95349 \\
\hline
\end{tabular}

Table IV shows the successful frame transmission rates within TXOP periods for all ACs in the WBAN/WLAN bridges and Regular WLAN nodes. The successful frame transmission probabilities for all ACs in the regular WLAN nodes are identical due to their equal data frame size. However, the probabilities in the bridges are different because the ACs' frame sizes could be unequal. The average payload sizes of the bridges' aggregated data frames are equal to $900 \mathrm{~B}$ for $\mathrm{AC}_{0}, 1100 \mathrm{~B}$ for $\mathrm{AC}_{1}, 400 \mathrm{~B}$ for $\mathrm{AC}_{2}$, and $600 \mathrm{~B}$ for $\mathrm{AC}_{3}$.

\section{CONCLUSION}

For transferring the medical data collected in the WBANs to the medical server we bridged the IEEE 802.15.6-based WBANs and the IEEE 802.11e EDCA WLAN and mapping 8 WBAN UPs into 4 WLAN ACs. Since the medical data has to pass through the WLAN, the WLAN priority differentiations and number of regular WLAN nodes considerably affect the healthcare network performance. In this paper, we investigated the impacts of AC differentiation by AIFS and CW on the WBANs' and WLAN's data traffic transmissions under low to medium traffic loads in the WBAN/WLAN bridged healthcare network. The results of this paper indicate that for a small number of regular nodes in the network the $\mathrm{AC}$ differentiation by AIFS and $\mathrm{CW}$ provide approximately identical results. However, the AC differentiation by AIFS significantly outperforms the AC differentiation by $\mathrm{CW}$ for the case when there are a large number of regular WLAN nodes with moderate traffic rate. Smaller contention window sizes would result in larger collision probability and would 


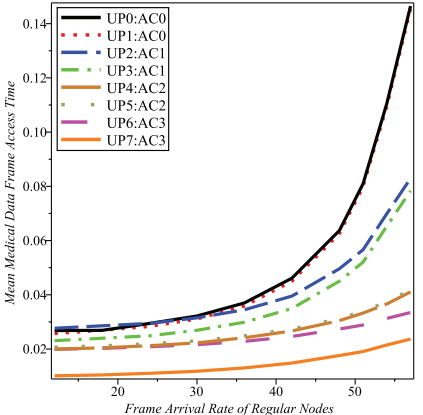

(a) 3 regular nodes, AIFS differentiation

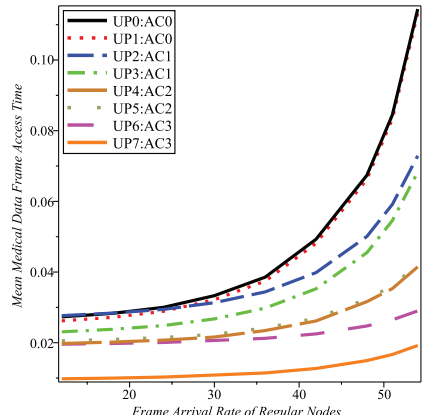

(b) 3 regular nodes, CW differentiation

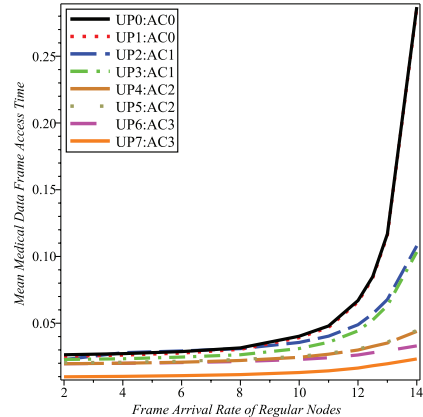

(c) 10 regular nodes, AIFS differentiation

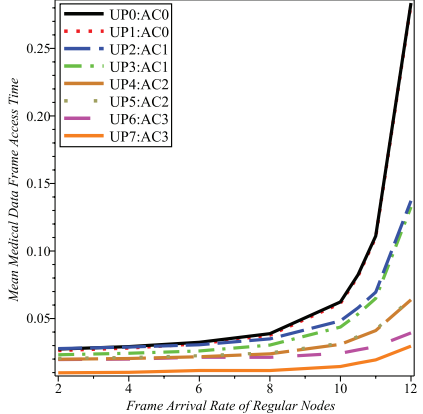

(d) 10 regular nodes, $\mathrm{CW}$ differentiation

Fig. 3. Mean medical data frame access time includes the medical data frame response time in WBAN and the aggregated frame response time in WLAN. $\mathrm{BER}=2 * 10^{-5}$ and TXOP $=5000 \mu \mathrm{sec}$.

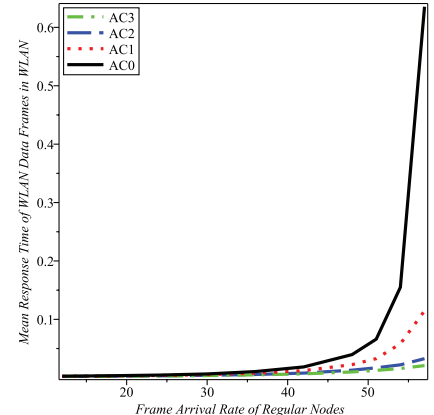

(a) 3 regular nodes, AIFS differentiation

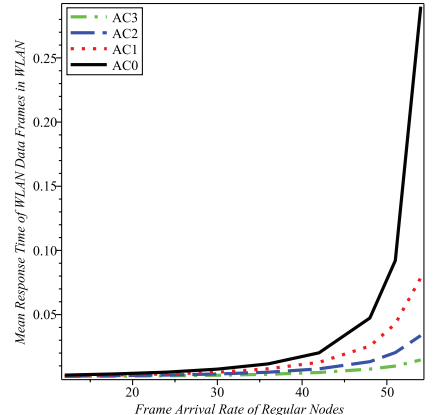

(b) 3 regular nodes, CW differentiation

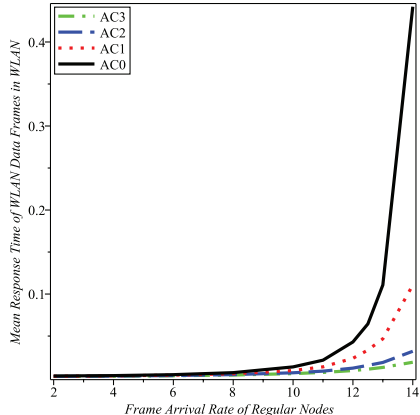

(c) 10 regular nodes, AIFS differentiation

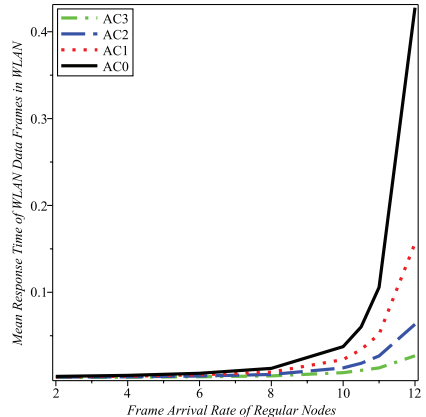

(d) 10 regular nodes, CW differentiation

Fig. 4. Mean response time of WLAN data frames in the WLAN. BER $=2 * 10^{-5}$ and TXOP $=5000 \mu$ sec.

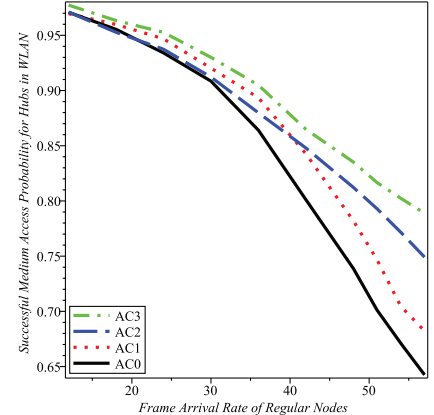

(a) 3 regular nodes, AIFS differentiation

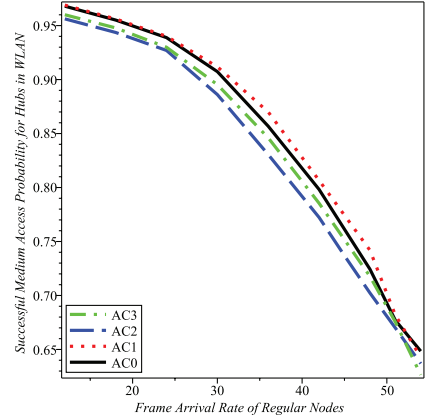

(b) 3 regular nodes, CW differentiation

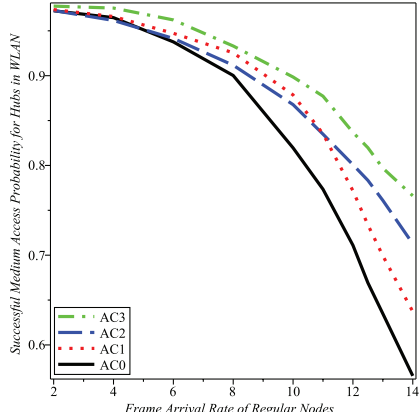

(c) 10 regular nodes, AIFS differentiation

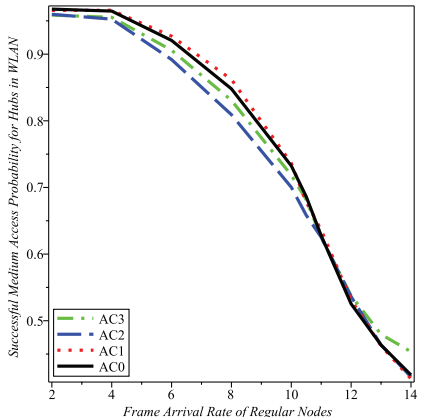

(d) 10 regular nodes, $\mathrm{CW}$ differentiation

Fig. 5. Successful medium access probability in the WLAN. BER $=2 * 10^{-5}$ and TXOP $=5000 \mu$ sec.

cause high contention in the WLAN with a large number of contending nodes. Therefore, because AIFS provides moderate AC differentiation, lower collision probabilities, and preserves the relative order of WBAN frame response times, it is desirable to be used for the differentiation in the WLAN.

\section{REFERENCES}

[1] Wireless Body Area Network Draft Standard, IEEE Std. 802.15.6, 2010.

[2] Wireless LAN Medium Access Control (MAC) and Physical Layer (PHY) Specification, IEEE Std. 802.11, 2007.
[3] S. Rashwand and J. Misic, "Performance evaluation of IEEE 802.15.6 under non-saturation condition," in Proc. the IEEE Global Telecommunications Conference (Globecom11), Houston, Texas, US., Dec. 2011.

[4] — " "IEEE 802.11e EDCA under bursty traffic - how much TXOP can improve performance," IEEE Transactions on Vehicular Technology (TVT), vol. 60, pp. 1099-1115, 2011.

[5] L. Kleinrock, Queuing Systems - Volume1: Theory. New York: John Wiley \& Sons, 1975

[6] S. Ullah and K. S. Kwak, "Throughput and delay limits of IEEE 802.15.6," in Proc. IEEE WCNC, Cancun, Mexico, Mar. 2011.

[7] F. Martelli, C. Buratti, and R. Verdone, "On the performance of an IEEE 802.15.6 wireless body area network," in Proc. IEEE European Wireless 


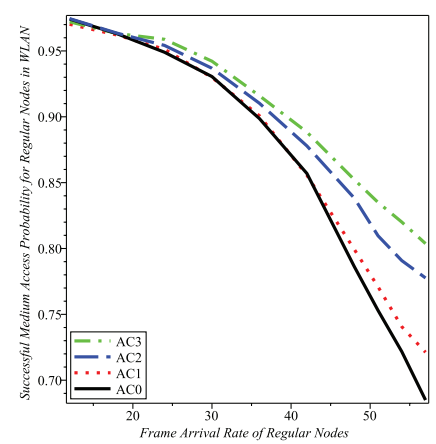

(a) 3 regular nodes, AIFS differentiation

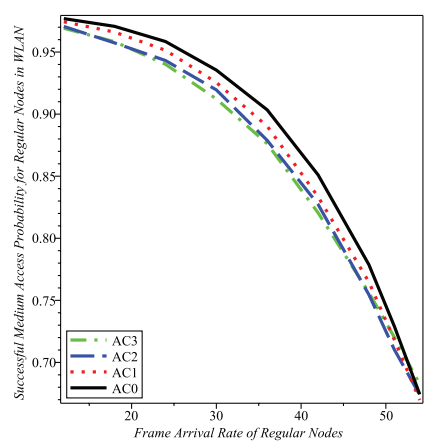

(b) 3 regular nodes, CW differentiation

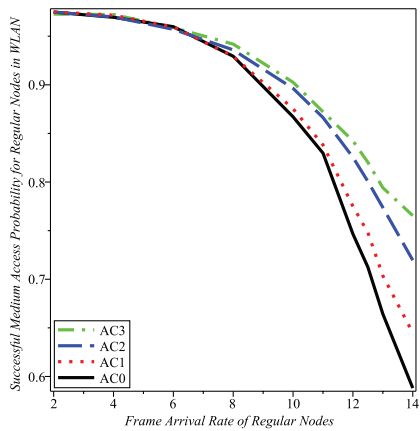

(c) 10 regular nodes, AIFS differentiation

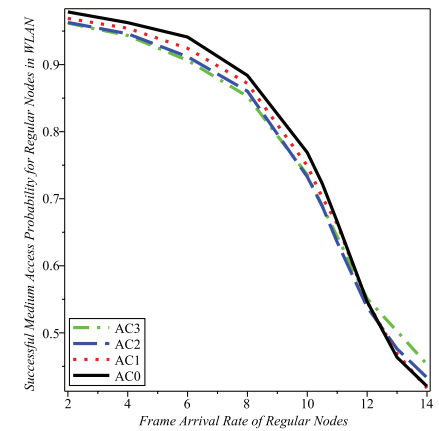

(d) 10 regular nodes, $\mathrm{CW}$ differentiation

Fig. 6. Successful medium access probability in the WLAN. BER $=2 * 10^{-5}$ and TXOP $=5000 \mu$ sec.

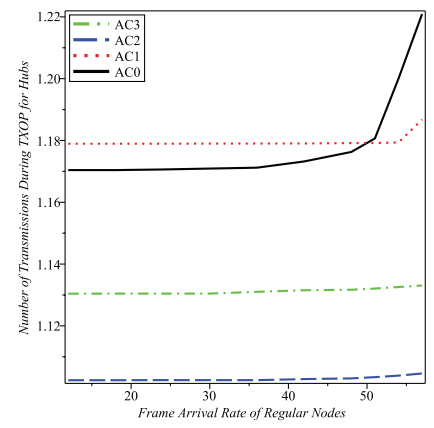

(a) 3 regular nodes, AIFS differentiation

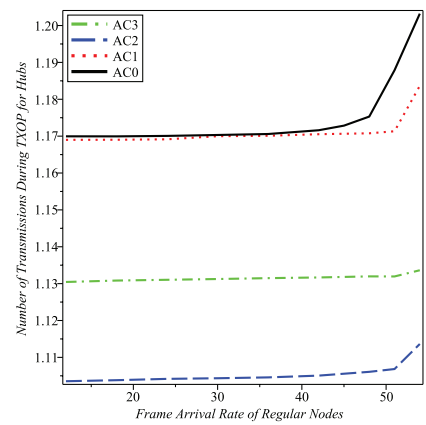

(b) 3 regular nodes, CW differentiation

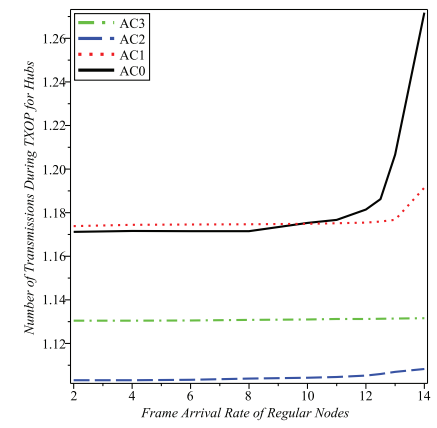

(c) 10 regular nodes, AIFS differentiation

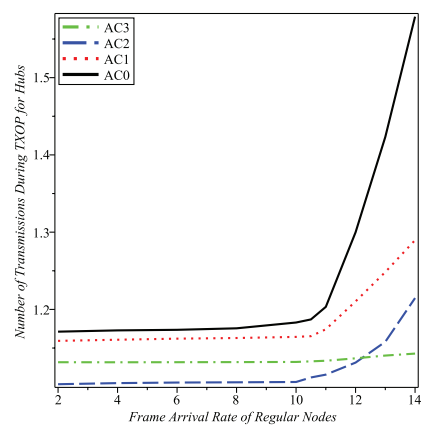

(d) 10 regular nodes, $\mathrm{CW}$ differentiation

Fig. 7. Mean number of successfully transmitted frames during a TXOP access for hubs in the WLAN. BER=2*10 ${ }^{-5}$ and TXOP $=5000 \mu$ sec.

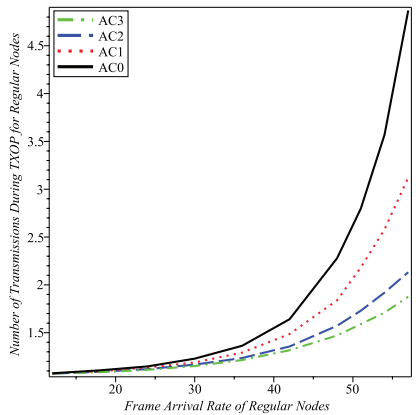

(a) 3 regular nodes, AIFS differentiation

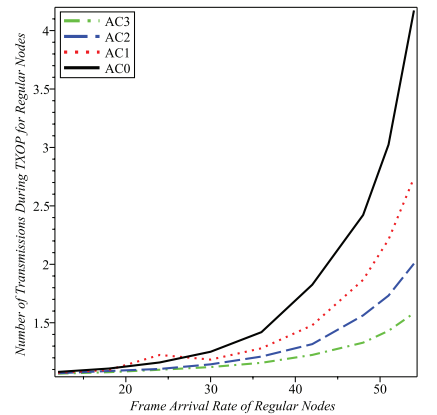

(b) 3 regular nodes, CW differentiation

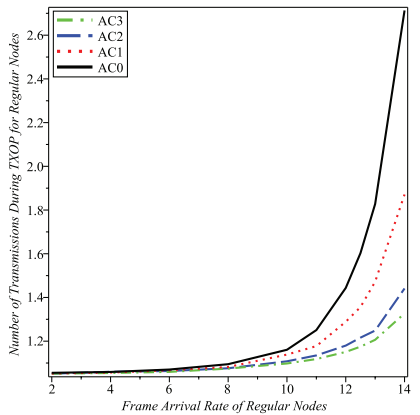

(c) 10 regular nodes, AIFS differentiation

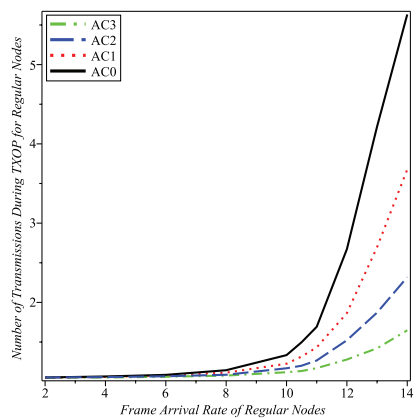

(d) 10 regular nodes, CW differentiation

Fig. 8. Mean number of transmitted frames during a TXOP access for regular nodes in the WLAN. BER $=2 * 10^{-5}$ and TXOP $=5000 \mu$ sec.

Conference, Vienna, Austria, 2011, pp. 1-6.

[8] O. M. F. Abu-Sharkh and A. H. Tewfik, "Toward accurate modeling of the ieee 802.11e EDCA under finite load and error-prone channel," IEEE Transactions on Wireless Communications, vol. 7, pp. 2560-2570, 2008.

[9] J. Y. Lee and H. S. Lee, "A performance analysis model for ieee 802.11e EDCA under saturation condition," IEEE Transactions on Communications, vol. 57, pp. 56-63, 2009.

[10] R. S. Uppal and S. Puri, "Performance and evaluation of IEEE 802.11e using QUALNET," International Journal on Computer Science and Engineering (IJCSE), vol. 3, pp. 1327-1332, 2011.

[11] S. Rashwand, J. Misic, and V. Misic, "MAC performance modeling of
IEEE 802.15.6-based WBANs over Rician-faded channels," in Proc. the IEEE International Conference on Communications (ICC12), Ottawa, Canada, 2012.

[12] (2010) Opnet modeler, opnet technologies, inc. Bethesda, MD. [Online]. Available: http://www.opnet.com/ 